\title{
NGC 6388 reloaded: some like it hot, but not too much. New constraints on the first-generation polluters ${ }^{\star}$
}

\author{
Eugenio Carretta and Angela Bragaglia
}

\begin{abstract}
INAF-Osservatorio di Astrofisica e Scienza dello Spazio di Bologna, Via Gobetti 93/3, 40129 Bologna, Italy e-mail: eugenio.carretta@inaf.it
\end{abstract}

Received 6 June 2019 / Accepted 25 June 2019

\begin{abstract}
Multiple stellar populations in globular clusters (GCs) are defined and recognized by their chemical signature. Second-generation stars show the effects of nucleosynthesis in the more massive stars of the earliest component that formed in the first star formation burst. High-temperature H-burning produces the whole pattern of (anti-) correlations in proton-capture elements that are widely found in GCs. However, it is still debated where this burning occurred. Here we introduce new powerful diagnostic plots to detect evidence (if any) of products from proton-capture reactions that occur at very high temperatures. To test these detectors of high-temperature H-burning plots, we show that stringent constraints can be placed on the temperature range of the first-generation polluters that contributed to shaping the chemistry of multiple stellar population in the massive bulge GC NGC 6388. Using the largest sample to date (185 stars) of giants with detailed abundance ratios in a single GC (except $\omega$ Cen), we may infer that the central temperature of part of the polluters must have been comprised between $\sim 100$ and $\sim 150 \mathrm{MK}$ if we consider hydrostatic H-burning in the core of massive stars. A much more narrow range (110-120 MK) is inferred if the polluters can be identified in massive asymptotic giant branch stars.
\end{abstract}

Key words. stars: abundances - stars: atmospheres - stars: Population II - globular clusters: individual: NGC 6388 globular clusters: general

\section{Introduction}

The multiple stellar populations in Galactic globular clusters (GCs) are characterized by their pattern of light elements (C, N, O, Na, Mg, Al, Si, and sometime heavier species such as $\mathrm{Ca}, \mathrm{Sc}$, and $\mathrm{K}$ ). One component, which is believed to be the first to be formed in a proto-GC, presents the same level of abundance ratios as the vast majority of metal-poor halo stars at the same metallicity. However, the so-called second generation (SG) shows alterations in the abundances of these elements that range from moderate to extreme. The network of correlations and anticorrelations found among these species represents an important clue for explaining the origin of multiple stellar populations because all the observed relations can be traced back to one single mechanism, H-burning.

Since the pioneering studies by Denisenkov (1989) and Langer et al. (1993), it was clear that proton-capture reactions in H-burning at high temperature could account for all the observed chemical modifications. The enhancement in the abundance ratios of a given element is accompanied (and explained) by the depletion of some other species that participate in the network of proton-capture reactions. The uncertainties related to the modeling of stellar structures and to the release of processed material into the intracluster medium currently hamper our understanding of the exact sites at which this burning occurred. Several candidate polluters were proposed, ranging

* Based on observations collected at ESO telescopes under programmes 073.D-0211, 073.D-0760, 381.D-0329, 095.D-0834, and 099.D-0047. from less massive (intermediate-mass asymptotic giant branch stars, AGB; Cottrell \& Da Costa 1981; Ventura et al. 2001) to fast-rotating massive stars (Decressin et al. 2007) or interacting massive binaries (de Mink et al. 2009) and to supermassive objects (Denissenkov \& Hartwick 2014).

Fortunately, the variety of nuclear reactions involved in this burning provides some clues because proton-capture on increasingly heavier nuclei must account for the increasing Coulomb barrier. As a consequence, increasingly higher temperatures are required to activate the relevant reactions, which thus provide ideal thermometers for probing the inner structure of the stellar sites at which nucleosynthesis occurred. Thus, fusion temperatures $\geq 40 \mathrm{MK}$ suffice to convert $\mathrm{O}$ into $\mathrm{N}$ and simultaneously produce $\mathrm{Na}$ from the $\mathrm{NeNa}$ cycle. This rather modest threshold explains why the $\mathrm{Na}-\mathrm{O}$ anticorrelation is so common in GCs that it was proposed to be considered the main signature of the presence of multiple populations, or in other words, the genuine essence of a GC (Carretta et al. 2010). The higher temperatures $(\geq 70 \mathrm{MK})$ that are required for the $\mathrm{MgAl}$ cycle to operate efficiently are apparently only possible in stars in a subset of GCs because the $\mathrm{Mg}$-Al anticorrelation was only found in massive and/or metal-poor GCs (Carretta et al. 2009a), as later confirmed by further studies (Mészáros et al. 2015; Pancino et al. 2017; Nataf et al. 2019). These are also the GCs where some overproduction of $\mathrm{Si}$ is observed (e.g., Carretta et al. 2009a), which indicates that temperatures in excess of $80-100 \mathrm{MK}$ were reached in the polluters (e.g., Prantzos et al. 2017). Finally, even higher temperatures >100-180 MK (Ventura et al. 2012; Prantzos et al. 2017) 


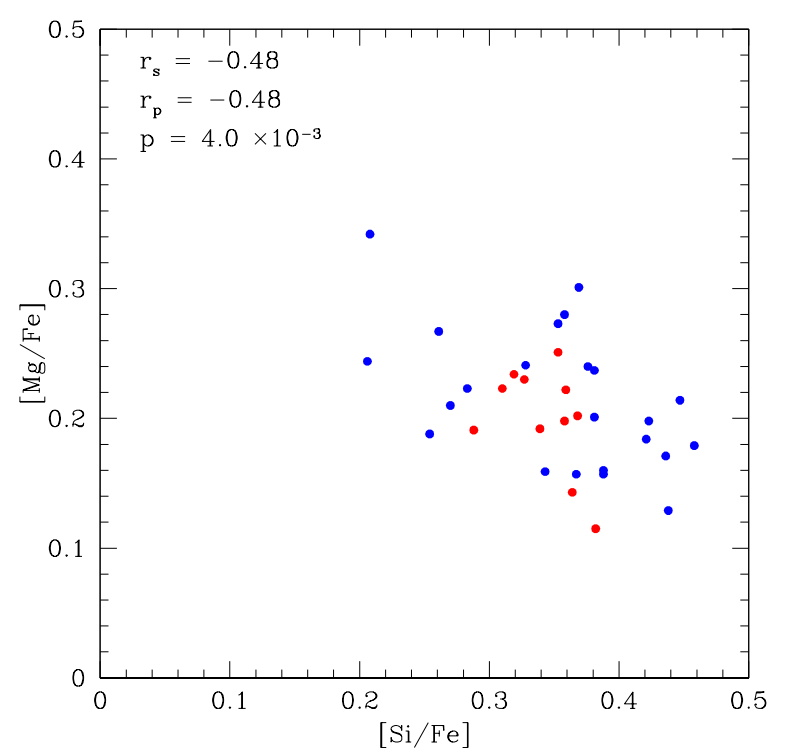

Fig. 1. Anticorrelation of $[\mathrm{Mg} / \mathrm{Fe}]$ and $[\mathrm{Si} / \mathrm{Fe}]$ abundance ratios in NGC 6388. Blue and red circles indicate stars from Paper I and the new stars analyzed in Carretta \& Bragaglia (in prep.), respectively. We list the Spearman rank correlation $\left(r_{\mathrm{S}}\right)$ coefficient, the Pearson linear correlation $\left(r_{\mathrm{P}}\right)$ coefficient, and its $p$-value.

are required to explain the $\mathrm{Mg}-\mathrm{K}$ and $\mathrm{Mg}-\mathrm{Sc}$ anticorrelations, including some of the heaviest proton-capture species, that are observed in some peculiar GCs: NGC 2808 (Mucciarelli et al. 2015; Carretta 2015) and NGC 2419 (Mucciarelli et al. 2012; Cohen \& Kirby 2012).

In this Letter we exploit these different thermometers with the aim to design new diagnostic plots that we call detectors of high-temperature (DOHT) H-burning products because they allow us to reveal the effects (if any) of reactions that were activated at unusually high temperatures that exceed the temperatures that typically occur in most cluster polluters. We test the DOHT plots by placing strong constraints on the temperature range of the putative polluters that shaped the chemical pattern of multiple populations in the massive GC NGC 6388.

Our data and analysis are briefly summarized in Sect. 2. We present our results in Sect. 3 and discuss them in Sect. 4.

\section{Dataset}

The GC NGC 6388 is a bulge cluster of high total mass, but only 37 stars with determined abundances of proton-capture elements were available (Carretta et al. 2007, 2009b) because a high field contamination affects this GC. This problem was recently overcome (Carretta \& Bragaglia 2018, hereinafter Paper I) by exploiting the large sample of stellar spectra in the cluster region that is present in the ESO archive, and also by newly granted observing time. Here we present results based on homogeneous abundances of $\mathrm{Mg}, \mathrm{Ca}$, and $\mathrm{Sc}$ of more than 180 giants. This is the largest sample of stars with a detailed abundance analysis in a GC (with the notable exception of $\omega$ Cen). This dataset was used to test the new DOHT plots, as shown in the next section.

We employed FLAMES (Fibre Large Array Multi Element Spectrograph, Pasquini et al. 2002) data. Our results are based on abundances that are partly derived from UVES spectra that we presented in Paper I (23 stars) and partly from new UVES spectra (11 stars). We also used 149 stars with abundances of $\mathrm{Fe}, \mathrm{Mg}, \mathrm{Ca}, \mathrm{Sc}$ from GIRAFFE spectra and the high-resolution setup HR13. We acquired new data with the ESO program

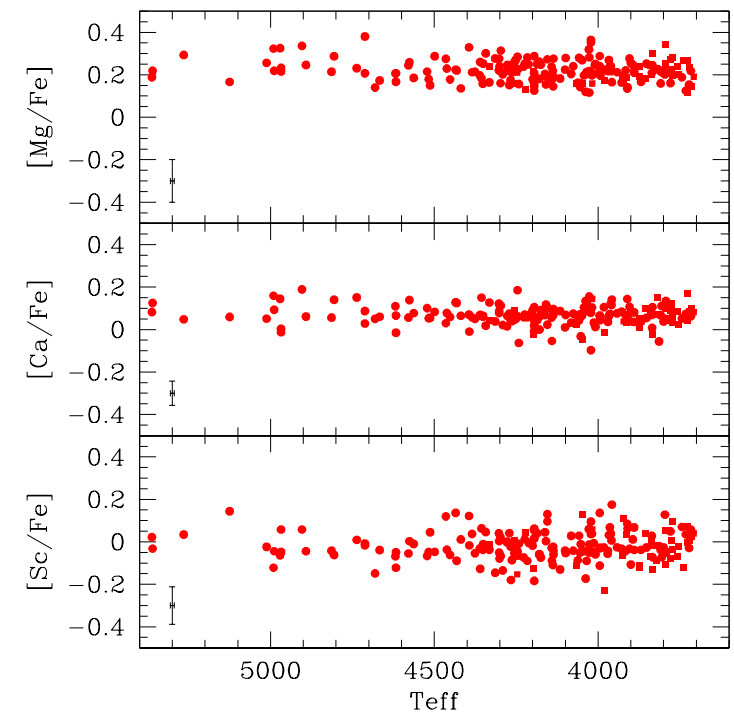

Fig. 2. Ratios $[\mathrm{Mg} / \mathrm{Fe}]$ (184 stars), $[\mathrm{Ca} / \mathrm{Fe}]$, and $[\mathrm{Sc} / \mathrm{Fe}]$ (185 stars) from FLAMES spectra in NGC 6388 (Carretta \& Bragaglia, in prep.) as a function of effective temperature. Internal error bars are shown in each panel.

099.D-0047 (PI Carretta) and used archival spectra of three programs: 381.D-0329(B), PI Lanzoni; 073.D-0760(A), PI Catelan; 095.D-0834(A), PI Henault-Brunet, together with the original data from our FLAMES survey (073.D-0211, PI Carretta). The detailed abundance analysis for all stars that we did not present in Paper I will be described in a forthcoming paper (Carretta \& Bragaglia, in prep.). However, the analysis closely follows our homogeneous procedures (see Carretta et al. 2009a,b for the UVES and GIRAFFE spectra, respectively) for deriving abundances and estimating star-to-star errors. The metallicity for NGC 6388 is $[\mathrm{Fe} / \mathrm{H}]=-0.480 \mathrm{dex}$ ( $\mathrm{rms}=0.045 \mathrm{dex}, 35$ stars), based on UVES spectra (Carretta \& Bragaglia, in prep.) and on the same method as we used to derive the metal abundance for NGC $2808([\mathrm{Fe} / \mathrm{H}]=-1.129 \mathrm{dex}, \mathrm{rms}=0.030 \mathrm{dex}, 31$ stars, Carretta 2015) that we use as a comparison in the next section.

\section{Temperature range of $F G$ polluters in NGC 6388}

The first constraint expands on the result we reported in Paper I and is based on abundances from UVES spectra. In Fig. 1 we show the Mg-Si anticorrelation in NGC 6388, which is found to be statistically very significant: the Pearson regression coefficient $r_{\mathrm{P}}$ is -0.48 (34 stars), with a two-tail probability to be a random result $p=4.0 \times 10^{-3}$.

This is clearly due to the well-known leakage from $\mathrm{Mg}$-Al cycle on ${ }^{28} \mathrm{Si}$ : when the ${ }^{27} \mathrm{Al}(p, \gamma){ }^{28} \mathrm{Si}$ reaction takes over ${ }^{27} \mathrm{Al}(p, \alpha){ }^{24} \mathrm{Mg}$, a certain amount of ${ }^{28} \mathrm{Si}$ is produced by protoncaptures (see Karakas \& Lattanzio 2003). This overproduction of Si typically occurs at temperatures $>100 \mathrm{MK}$, even if the leakage from the $\mathrm{Mg}$-Al cycle already starts at about $65 \mathrm{MK}$ (Arnould et al. 1999). The first constraint then stems from these observations, which are strengthened and well assessed by the analysis of 11 new red giants with UVES spectra in Carretta \& Bragaglia (in prep.; red points in Fig. 1).

A second limit to the temperature range in first-generation (FG) polluters that is active at cluster formation may be provided by the abundances of $\mathrm{Mg}, \mathrm{Ca}$, and $\mathrm{Sc}$, which are available for 184, 185, and 185 stars, respectively, in NGC 6388 (Carretta \& Bragaglia, in prep.). The abundance ratios are plotted in Fig. 2 as a function of the effective temperatures. 

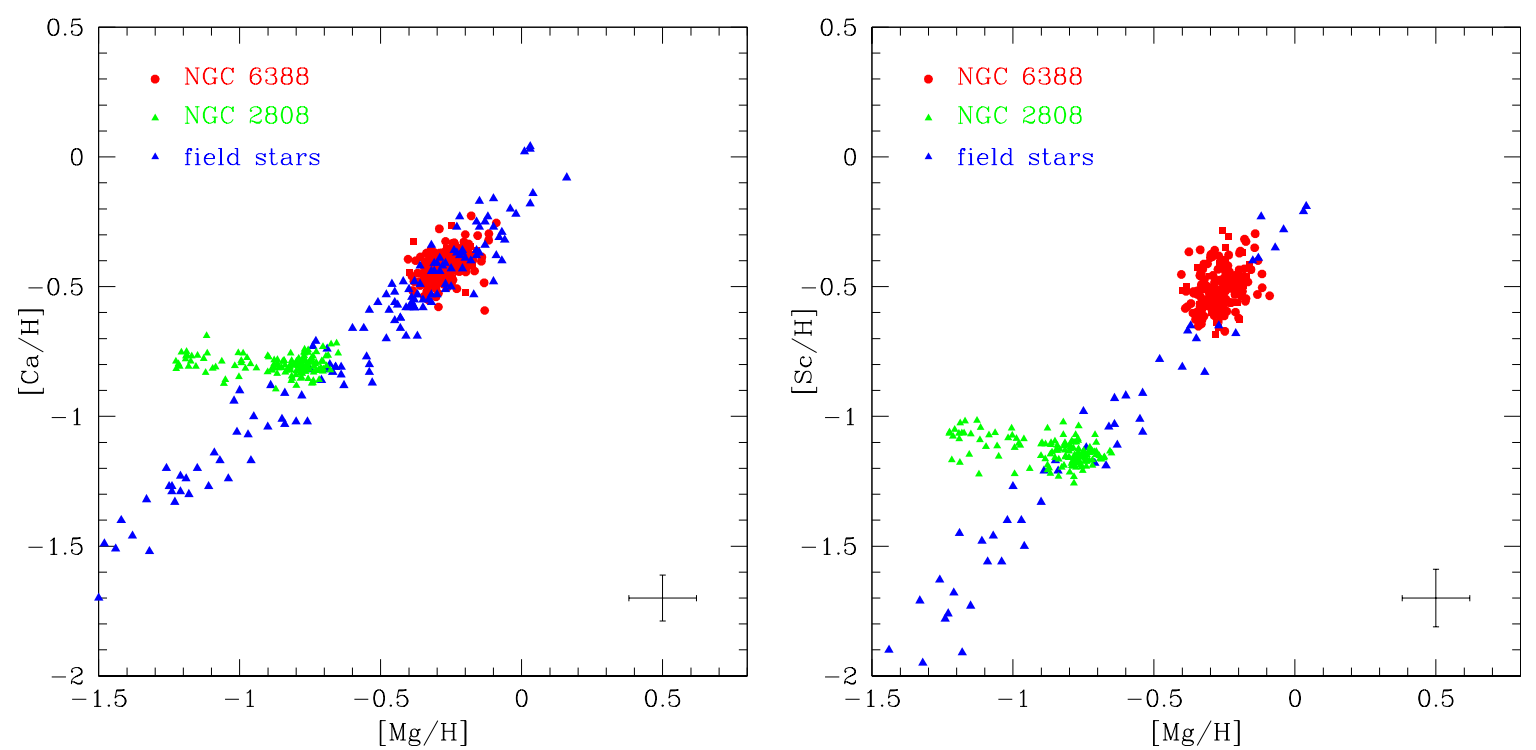

Fig. 3. Ratios $[\mathrm{Ca} / \mathrm{H}]$ (left panel) and $[\mathrm{Sc} / \mathrm{H}]$ (right panel) as a function of $[\mathrm{Mg} / \mathrm{H}]$ for giants in $\mathrm{NGC} 6388$ (red points), superimposed on field stars from Gratton et al. (2003; blue triangles). Green triangles are giants in NGC 2808 from Carretta \& Bragaglia (in prep.). Internal star-to-star errors refer to NGC 6388.

Star-to-star error bars are also shown and estimated (as described in Carretta et al. 2009b) as $0.100 \mathrm{dex}, 0.057 \mathrm{dex}$, and $0.088 \mathrm{dex}$ for $[\mathrm{Mg} / \mathrm{Fe}],[\mathrm{Ca} / \mathrm{Fe}]$, and $[\mathrm{Sc} / \mathrm{Fe}]$, respectively.

The constraint on the H-burning temperature in the stellar sites that forged the proton-capture elements that in turn form the multiple populations in NGC 6388 may be fully appreciated from the two panels in Fig. 3, which we call DOHT plots. In this figure we used field stars from Gratton et al. (2003) as reference because most of the field stars only show the chemical pattern derived from the supernovae $(\mathrm{SNe})$ nucleosynthesis, with no trace of the peculiar proton-capture nucleosynthesis, which is restricted to the dense environment of GCs (e.g., Gratton et al. 2000; Smith \& Martell 2003).

The distribution of stars of NGC 6388 (red points) in the $\mathrm{Ca}-\mathrm{Mg}$ and $\mathrm{Sc}-\mathrm{Mg}$ planes almost perfectly matches that of field stars: $\mathrm{Ca}$ and $\mathrm{Sc}$ abundances in this GC are not modified by the action of proton-capture reactions. Conversely, the observations present a different scenario in NGC 2808, which is a GC that is slighly less massive than NGC 6388 (total absolute magnitude $M_{V}=-9.39$ and $M_{V}=-9.41 \mathrm{mag}$, respectively: Harris 1996 , online version 2010). The same abundances are plotted (green triangles) in the two panels of Fig. 3, and all elemental ratios are obtained with extremely homogeneous procedures that were also adopted for NGC 6388. The pattern that is observable in NGC 2808 is strikingly different: the abundances of both $\mathrm{Ca}$ and $\mathrm{Sc}$ are anticorrelated to the abundances of $\mathrm{Mg}$. Both these relations are found to be statistically very significant (Carretta 2015). This behavior was attributed to the same process of protoncapture at very high temperature (above $100 \mathrm{MK}$ ) that was postulated to act in GCs such as NGC 2419 to produce potassium from proton-capture on ${ }^{36} \mathrm{Ar}$ nuclei (Ventura et al. 2012). The observations in NGC 2808 are explained with this high-temperature process, where the formation of proton-rich species is shifted toward heavier nuclei. The effects are obviously more marked on $\mathrm{Sc}$ (and $\mathrm{K}$ ) than on $\mathrm{Ca}$ because $\mathrm{Ca}$ is much more abundant. The inference for the case of NGC 6388 is straightforward: the H-burning in the FG polluters in this GC was not able to reach temperatures that were high enough to substantially modify the abundances of $\mathrm{Ca}$ and $\mathrm{Sc}$ with respect to the levels from $\mathrm{SNe}$ nucleosynthesis.

\section{Discussion and conclusions}

Summarizing the observational evidence found in NGC 6388, we note (i) that the overproduction of $\mathrm{Si}$ is likely due to the increased leakage from the $\mathrm{Mg}-\mathrm{Al}$ cycle (Paper I and this Letter, Fig. 1), and (ii) the absence of significant $\mathrm{Ca}-\mathrm{Mg}$ and $\mathrm{Sc}-\mathrm{Mg}$ anticorrelations in the new diagnostic plot we introduced here (Fig. 3).

Prantzos et al. (2017) made extensive H-burning nucleosynthesis calculations in the range of temperature and density that is suitable to hydrostatic burning in the H-shell of AGB stars or in the cores of massive and supermassive stars. After accounting for the minimum dilution factor that is required to reproduce the most extreme $\mathrm{O}$ abundances in NGC 2808, they found that a limited temperature range $(70-80 \mathrm{MK})$ is able to reproduce all the extreme observed values of $\mathrm{O}, \mathrm{Na}, \mathrm{Mg}$, and $\mathrm{Al}$ in this cluster. The high extreme of this range also accounts for the observed Si abundances in NGC 2808. Only the burning temperature required for $\mathrm{K}$ production exceeds this range because the extreme K abundances in NGC 2808 are only reproduced for $T \sim 180 \mathrm{MK}$. A similar discussion can be found in D'Antona et al. (2016).

In NGC 6388 we do not (yet) have determinations of $[\mathrm{K} / \mathrm{Fe}]$, but we can address the production of the heavy protoncapture elements by considering $\mathrm{Ca}$ and $\mathrm{Sc}$, which have similar atomic numbers, whose abundances are also clearly altered by $\mathrm{H}$-burning in FG polluters of NGC 2808 (Carretta 2015). At odds with the latter case, we here found from the DOHT plots that the distribution of Ca and Sc in NGC 6388 closely follows that of field stars at similar metallicity. Conversely, we see robust evidence that $\mathrm{Si}$ is altered (Paper I for the UVES sample and Carretta \& Bragaglia (in prep.) for the GIRAFFE sample), which implies temperatures higher than $100 \mathrm{MK}$. Because heavier elements (such as Ar and $\mathrm{K}$ ) do not start to be affected by nuclear burning before $T \sim 150 \mathrm{MK}$ (Prantzos et al. 2017), our results here allow us to pinpoint a rather limited range of temperature $(\sim 100-150 \mathrm{MK})$ in which the H-burning in the putative FG polluters occurred. An even narrower range can be estimated if the polluters can be identified in massive AGB stars. In Fig. 1 of D'Antona et al. (2016), at metallicities corresponding to that of 
NGC 6388, the maximum temperature $T_{\mathrm{HBB}}$ at the bottom of the convective envelope for masses evolving in AGB exceeds the temperature that is necessary to produce $\mathrm{Si}$, but never reaches the temperature that is required to process Ar into $\mathrm{K}$ or heavier species. In this case, a range 110-120 MK may explain the observed abundances in NGC 6388.

However, the limited sample of stars available in Paper I already indicated that a single source of pollution is probably not enough to fit all the multi-element observations with a dilution model in NGC 6388. More stringent conclusions will be drawn when the full set of light elements $(\mathrm{O}, \mathrm{Na}, \mathrm{Mg}, \mathrm{Al}, \mathrm{Si}$, with $\mathrm{Ca}$ and $\mathrm{Sc}$ as the heaviest extreme) will be available.

This Letter highlights an application with which the temperature range can be inferred at which past polluters were active. It also illustrates the precision that might be achieved with the constraints that are potentially available from large samples with a complete set of homogeneous abundances of proton-capture elements from spectroscopy. The new diagnostic DOHT plots seem to be a powerful tool for determining the existence of products from nuclear processing at very high temperatures. Only a few GCs in which this very advanced H-burning operated are currently known, but their census is growing. Among these GCs we may number NGC 2808 (Carretta 2015), NGC 2419 (Mucciarelli et al. 2012; Cohen \& Kirby 2012), and NGC 4833 (Carretta \& Bragaglia, in prep.).

Datasets like the one used here and in the forthcoming paper can be used by theoreticians to test and fine-tune both models and derived scenarios up to moderately high metal abundances. A set of calculations for NGC 6388, like those presented by D'Antona et al. (2016) and Prantzos et al. (2017) for NGC 2808 and Prantzos et al. (2007) for NGC 6752, would be very welcome, especially because the abundances in these three GCs, which span 1 dex in $[\mathrm{Fe} / \mathrm{H}]$, are obtained in a very homogeneous way.
Acknowledgements. We thank Raffaele Gratton for useful discussions. This research has made use of the services of the ESO Science Archive Facility, of the SIMBAD database (in particular Vizier), operated at CDS, Strasbourg, France, and of the NASA Astrophysical Data System.

\section{References}

Arnould, M., Goriely, S., \& Jorissen, A. 1999, A\&A, 347, 572 Carretta, E. 2015, ApJ, 810, 148

Carretta, E., \& Bragaglia, A. 2018, A\&A, 614, A109 (Paper I) Carretta, E., Bragaglia, A., Gratton, R. G., et al. 2007, A\&A, 464, 967

Carretta, E., Bragaglia, A., Gratton, R. G., \& Lucatello, S. 2009a, A\&A, 505, 139

Carretta, E., Bragaglia, A., Gratton, R. G., et al. 2009b, A\&A, 505, 117

Carretta, E., Bragaglia, A., Gratton, R. G., et al. 2010, A\&A, 516, A55

Cohen, J. G., \& Kirby, E. N. 2012, ApJ, 760, 86

Cottrell, P. L., \& Da Costa, G. S. 1981, ApJ, 245, L79

D’Antona, F., Vesperini, E., D'Ercole, A., et al. 2016, MNRAS, 458, 2122

de Mink, S. E., Pols, O. R., Langer, N., \& Izzard, R. G. 2009, A\&A, 507, L1

Decressin, T., Meynet, G., Charbonnel, C., et al. 2007, A\&A, 464, 1029

Denisenkov, P. A., \& Denisenkova, S. N. 1989, A. Tsir., 1538, 11

Denissenkov, P. A., \& Hartwick, F. D. A. 2014, MNRAS, 437, L21

Gratton, R. G., Sneden, C., Carretta, E., \& Bragaglia, A. 2000, A\&A, 354, 169

Gratton, R. G., Carretta, E., Claudi, R., Lucatello, S., \& Barbieri, M. 2003, A\&A, 404, 187

Harris, W. E. 1996, AJ, 112, 1487

Karakas, A. I., \& Lattanzio, J. C. 2003, PASA, 20, 279

Langer, G. E., Hoffman, R., \& Sneden, C. 1993, PASP, 105, 301

Mészáros, S., Martell, S. L., Shetrone, M., et al. 2015, AJ, 149, 153

Mucciarelli, A., Bellazzini, M., Ibata, R., et al. 2012, MNRAS, 426, 2889

Mucciarelli, A., Bellazzini, M., Merle, T., et al. 2015, ApJ, 801, 68

Nataf, D. M., Wyse, R., Schiavon, R. P., et al. 2019, AJ, 158, 14

Pancino, E., Romano, D., Tang, B., et al. 2017, A\&A, 601, A112

Pasquini, L., Avila, G., Blecha, A., et al. 2002, The Messenger, 110, 1

Prantzos, N., Charbonnel, C., \& Iliadis, C. 2007, A\&A, 470, 179

Prantzos, N., Charbonnel, C., \& Iliadis, C. 2017, A\&A, 608, A28

Smith, G. H., \& Martell, S. L. 2003, PASP, 115, 1211

Ventura, P., D’Antona, F., Mazzitelli, I., \& Gratton, R. 2001, ApJ, 550, L65

Ventura, P., D’Antona, F., Di Criscienzo, M., et al. 2012, ApJ, 761, L30 